\title{
Rare angiosarcoma of inferior turbinate; A case report and literature review
}

\author{
Mohd Eksan Sairin ${ }^{1 *}$, Noorizan Yahya ${ }^{1}$, Chew Yok Kuan ${ }^{1}$, Mohamad Khir Abdullah ${ }^{1}$, Salina \\ Husain $^{2}$ \\ ${ }^{1}$ Department of Otorbinolaryngology, Head and Neck Surgery, Hospital Pakar Sultanah Fatimah, Johor, Malaysia \\ ${ }^{2}$ Department of Otorbinolaryngology, Head and Neck Surgery, UKM Medical Centre, Kuala Lumpur, Malaysia
}

\begin{abstract}
Angiosarcoma is a rare soft-tissue sarcoma. It is an aggressive, malignant endothelial cell tumour of vascular or lymphatic in origin. Angiosarcoma accounts for $2 \%$ of all sarcomas and over half of it occurred in head and neck region. Its treatment is challenging with a poor prognosis. We presented a case of angiosarcoma of inferior turbinate occurring in a 73 year-old man.
\end{abstract}

Key Words: Angiosarcoma; inferior turbinate

\section{Introduction}

Angiosarcoma is a rare malignant endothelial cell tumour of vascular or lymphatic in origin (1). It is commonly seen in the skin and superficial soft tissues of the head and neck (2,3). Despite this fact, sinonasal angiosarcoma is extremely rare and accounts for less than $0.1 \%$ of all sinonasal tract malignancy (3). Epistaxis and nasal obstruction was the most frequently identified symptoms in sinonasal tract angiosarcoma (3) and surgical resection with radiation and/or chemotherapy is the treatment of choice $(1,3)$.

\section{Case report}

A 73-year-old gentleman presented with left neck swelling for 2 weeks duration. There were no ear, nose or throat symptoms. He had no history of fever, loss of weight and appetite. There was $1 \mathrm{~cm}$ x $1 \mathrm{~cm}$ firm, non-inflammed and mobile mass over the left posterior triangle of the neck. Nasoendoscopic examination revealed a friable mass arising from posterior end of left inferior turbinate. A fine needle aspiration cytology of the left neck swelling showed atypical cell with a suspicion of malignancy. Computed tomography scan of neck and paranasal sinuses showed an enhancing lobulated mass at the posterior aspect of left inferior turbinate. Histopathological examination of the mass revealed a vascular tumour in favour of angiosarcoma (FNCLCC
Grade 3). Patient was opted for surgery; however, he refused for any intervention including surgery, radiotherapy or chemotherapy. After 6 months follow up, patient remained well. The neck and nasal masses were not increasing in size.

\section{Discussion}

Angiosarcoma accounts for $2 \%$ of all sarcomas $(1,3)$ and over half of it occurred in head and neck, usually involving skin and superficial soft tissue. Angiosarcomas were subdivided into cutaneous angiosarcoma, lymphedema-associated angiosarcoma, radiation-induced angiosarcoma, primary-breast angiosarcoma and soft-tissue angiosarcoma (1). Most angiosarcomas arise sporadically as primary neoplasms. However, it can arise as secondary to pre-existing benign vascular lesion, chronic lymphedema or previous irradiation (1). Its occurrence in the sinonasal tract is less than $0.1 \%(3)$.

The tumour behaviour and clinical characteristics might depend on the site of origin. Epistaxis and nasal obstruction was the most frequently identified symptoms in sinonasal tract angiosarcoma (3). Patient also may present with nasal discharge, enlarging nasal mass, sinusitis, epiphora, diplopia, ptosis or headache. Its insidious presentation and multifocal nature are also well described (2). Interestingly, our patient had no nasal symptoms and only presented with unilateral neck swelling. The inferior turbinate

${ }^{*}$ Corresponding Author: Mohd Sairin Eksan MD, Department of Otorhinolaryngology, Head and Neck Surgery, Hospital Pakar Sultanah Fatimah, Jalan Salleh, 84000 Muar, Johor, Malaysia, Tel: +6069521901 Ext 119 or +60122356680, E-mail: eksansairin@yahoo.com Received: 01.07.2015, Accepted: 15.07.2016 
mass incidentally noted on nasoendoscopic examination. Its insidious onset with absence of local symptoms may lead to delay presentation and poorer prognosis. The differential diagnosis for sinonasal angiosarcoma includes granulation tissue, Masson's disease, hemangioma, juvenile nasopharyngeal angiofibroma, epitheloid hemangioma, glomangiopericytoma, Kaposi sarcoma and mucosal melanoma (3). Histologically, angiosarcoma can vary both within and between cases. Abnormal, pleomorphic, malignant endothelial cells are the hallmark of angiosarcoma and it can be rounded, polygonal or fusiform and can have an epitheloid appearance (1) (Figure 1). Immunohistochemical studies are important in confirming the diagnosis. It typically expresses endothelial markers including von

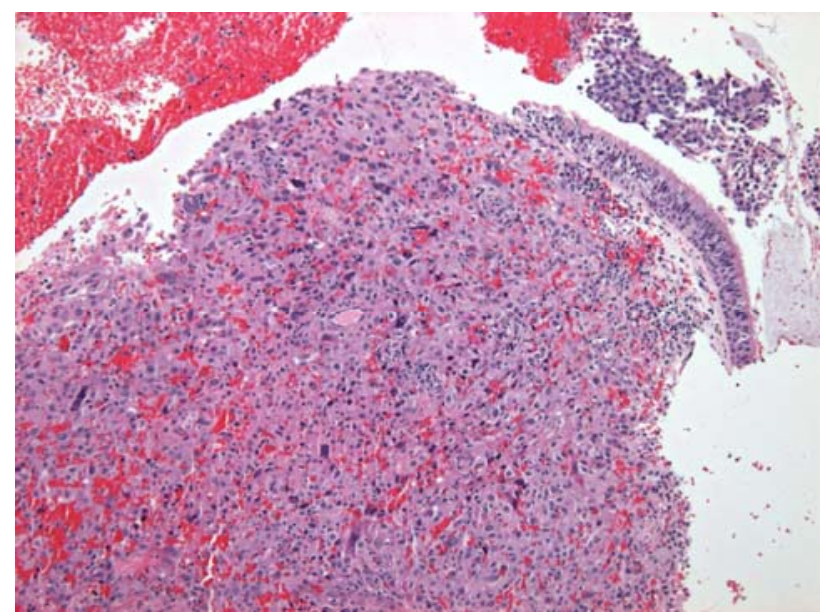

Fig. 1. The underlying stroma show anastomosing vascular channels lined by atypical epitheloid and spindle tumour cells with area of hemorrhage. Tumour cells display markedly pleomorphic nuclei, visible nucleoli and ill-defined eosinophilic cytoplasm.
Willebrand factor, CD34, CD31, Ulex europaeus agglutinin 1, and vascular endothelial growth factor (VEGF) and immunoreactive with vimentin, CD34, CD31, and Factor VIII-RA (3) (Figure 2 and 3 ).

The size of the primary lesion and presence of distant metastasis were the most useful determinant of treatment options. Surgical resection with radiation and/or chemotherapy is the treatment of choice in head and neck sarcomas $(1,3)$. Radical surgery with complete resection and adjuvant radiotherapy with large doses and wide treatment field was recommended for local disease whereas cytotoxic chemotherapy was the primary treatment for metastatic angiosarcoma (1). Successful management of sinonasal malignant neoplasms remains a significant surgical challenge.

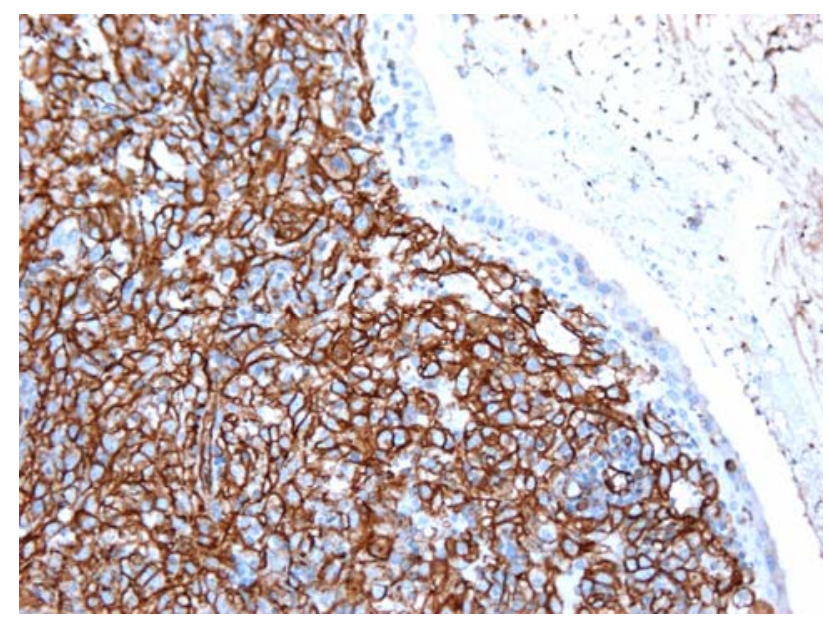

Fig. 2. Immunohistochemical staining: CD31 positive.

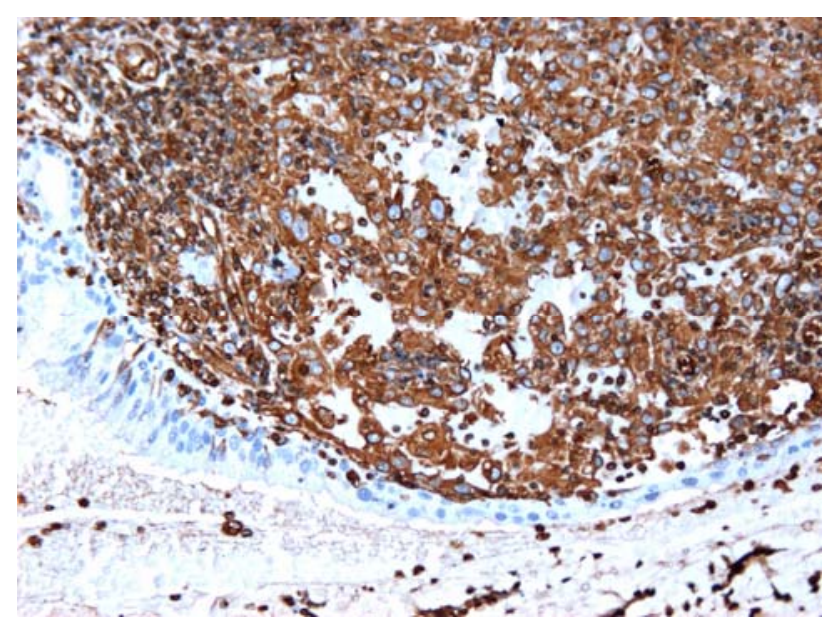

Fig. 3. Immunohistochemical stain: Vimentin positive.

East J Med Volume:22, Number:1, January-March/2017 
Evidence had supported the efficacy of endoscopic approach for definitive tumor extirpation for selected sinonasal malignancy and the benefits of the endoscopic techniques as a viable alternative to the traditional open approaches (4).

The primary soft tissue sarcoma was associated with 50\%-60\% 5-year survival but in general, angiosarcoma had an overall 5 year survival of about 35\% (1). Poor prognosis could be contributed by high grade tumour, large tumour $(>5 \mathrm{~cm})$, old age, metastatic disease at presentation and poor patient performance status (1). The common site for metastasis of sinonasal angiosarcoma includes lung, liver, spleen and bone marrow (3). There was no accepted staging for sinonasal tract sarcoma. Lymph node and distant metastasis was extremely rare at initial presentation (3). However, our case presented with a main complaint of neck swelling at presentation with a histopathology confirmation of lymph node metastasis.

In conclusion, angiosarcoma of sinonasal tract is a rare tumour, thus making an early diagnosis was challenging. Therefore, high index of suspicion with early histopathological examination and immunohistochemical studies could help in making an early diagnosis. Hence, the appropriate intervention can be commenced with the hope of better prognosis.

\section{References}

1. Young RJ, Brown NJ, Reed MW, Hughes D, Woll PJ. Angiosarcoma. Lancet Oncol 2010; 11: 983991.

2. Aust MR, Olsen KD, Lewis JE, Nascimento AG, Meland NB, Foote RL, Suman VJ. Angiosarcomas of the head and neck: clinical and pathologic characteristics. Ann Otol Rhinol Laryngol 1997; 106: 943-951.

3. Nelson BL, Thompson LD. Sinonasal tract angiosarcoma: a clinicopathologic and immunophenotypic study of 10 cases with a review of the literature. Head Neck Pathol 2007; 1: $1-12$.

4. Luong A, Citardi MJ, Batra PS. Management of sinonasal malignant neoplasms: defining the role of endoscopy. Am J Rhinol Allergy 2010; 24: 150155. 